ARTICLE

DOI: $10.1038 / \mathrm{s} 41467-018-02933-6$

\title{
Selective control of fcc and hcp crystal structures in Au-Ru solid-solution alloy nanoparticles
}

\author{
Quan Zhang ${ }^{1}$, Kohei Kusada ${ }^{1}$, Dongshuang $\mathrm{Wu}^{1}$, Tomokazu Yamamoto ${ }^{2,3}$, Takaaki Toriyama ${ }^{3}$, \\ Syo Matsumura ${ }^{2,3,4}$, Shogo Kawaguchi ${ }^{5}$, Yoshiki Kubota ${ }^{6}$ \& Hiroshi Kitagawa (1) 1,4
}

Binary solid-solution alloys generally adopt one of three principal crystal lattices-bodycentred cubic (bcc), hexagonal close-packed (hcp) or face-centred cubic (fcc) structures-in which the structure is dominated by constituent elements and compositions. Therefore, it is a significant challenge to selectively control the crystal structure in alloys with a certain composition. Here, we propose an approach for the selective control of the crystal structure in solid-solution alloys by using a chemical reduction method. By precisely tuning the reduction speed of the metal precursors, we selectively control the crystal structure of alloy nanoparticles, and are able to selectively synthesize fcc and hcp AuRu $\mathrm{All}_{3}$ ay nanoparticles at ambient conditions. This approach enables us to design alloy nanomaterials with the desired crystal structures to create innovative chemical and physical properties.

\footnotetext{
${ }^{1}$ Division of Chemistry, Graduate School of Science, Kyoto University, Kitashirakawa-Oiwakecho, Sakyo-ku, Kyoto 606-8502, Japan. ${ }^{2}$ Department of Applied Quantum Physics and Nuclear Engineering, Kyushu University, 744 Motooka, Nishi-ku, Fukuoka 819-0395, Japan. ${ }^{3}$ The Ultramicroscopy Research Center, Kyushu University, Motooka 744, Nishi-ku, Fukuoka 819-0395, Japan. ${ }^{4}$ INAMORI Frontier Research Center, Kyushu University, Motooka 744, Nishi-ku, Fukuoka 819-0395, Japan. ${ }^{5}$ Japan Synchrotron Radiation Research Insitute (JASRI), SPring-8, 1-1-1 Kouto, Sayo-cho, Sayo-gun, Hyogo 679-5198, Japan.

${ }^{6}$ Department of Physical Science, Graduate School of Science, Osaka Prefecture University, Sakai, Osaka 599-8531, Japan. Correspondence and requests for materials should be addressed to K.K. (email: kusada@kuchem.kyoto-u.ac.jp) or to H.K. (email: kitagawa@kuchem.kyoto-u.ac.jp)
} 
$\mathrm{T}$ he crystal structure is one of the most dominant factors that strongly affect the properties of an alloy, because the electronic structure changes drastically with the crystal structure $^{1,2}$. A solid-solution alloy, in which the constituents are randomly mixed at the atomic scale, generally adopts one of three principal crystal lattice forms: body-centred cubic (bcc), hexagonal close-packed (hcp) and face-centred cubic (fcc) structures. However, once its constituent element and composition are fixed, the crystal structure of solid-solution alloy is uniquely determined $^{3-5}$. Therefore, it is difficult to change the crystal structure of a solid-solution alloy at a certain composition.

Very recently, the crystal structure control of monometallic nanoparticles (NPs) at mild conditions has been reported ${ }^{6-10}$. For example, although bulk gold ( $\mathrm{Au}$ ) adopts only a fcc structure, hcp $\mathrm{Au}$ was obtained as an ultrathin nanosheet with a thickness of a few nanometres 8,9 . Furthermore, ruthenium $(\mathrm{Ru}) \mathrm{NPs}$ with a fcc structure were discovered and exhibited enhanced catalytic properties, even though $\mathrm{Ru}$ usually adopts only an hcp structure $^{10}$. These reports opened a new way to design novel monometallic NPs. However, to date, a rational approach to control the crystal structure of metals is not well developed. Given this situation, the rational control of the crystal structure of an alloy system is also very attractive and challenging.

Here, we propose an approach for the selective control of the crystal structure of solid-solution alloys at a certain composition using the chemical reduction method. If the alloy consists of elements whose bulk metals adopt different crystal structures, such as fcc and hcp, the fcc or hcp structure is thermodynamically and uniquely determined by its composition. However, for NPs, since the crystal structure would be strongly governed by the crystal nucleus during the alloy formation process, it is possible to selectively control its crystal structure by modifying the conditions of the crystal formation process. The crystal structure of binary alloy NPs could be dominated by the structure of the nuclei that are formed from one of the constituent metal ions, which start to be reduced slightly earlier than the other during the alloy formation process. By finely tuning the reduction speed of the metal precursors, which is one of the advantages of the chemical reduction method, we achieve the selective control of the crystal structure. In this paper, a $\mathrm{Au}-\mathrm{Ru}$ system whose parent metals, $\mathrm{Au}$ and $\mathrm{Ru}$, adopt the fcc and hcp structures, respectively, is chosen as the target demonstration for the selective control of the crystal structure, even though they cannot form a solidsolution phase, even in the liquid phase ${ }^{11}$. We succeed in selectively synthesizing fcc and hcp AuRu solid-solution alloy NPs at the $\mathrm{Au} / \mathrm{Ru}$ ratio of 1:3. The structures of the obtained NPs are investigated using synchrotron powder X-ray diffraction (XRD) analysis and atomic resolution scanning transmission electron microscopy (STEM). In addition, we discuss the mechanism of crystal structure control using the results of ultraviolet-visible (UV-vis) spectral and electrochemical analyses.

\section{Results}

Syntheses of $\mathrm{AuRu}_{3}$ NPs with fcc and hcp structures. The $\mathrm{AuRu}_{3}$ solid-solution NPs were synthesized using a polyol reduction method. For the synthesis of the fcc alloy NPs,
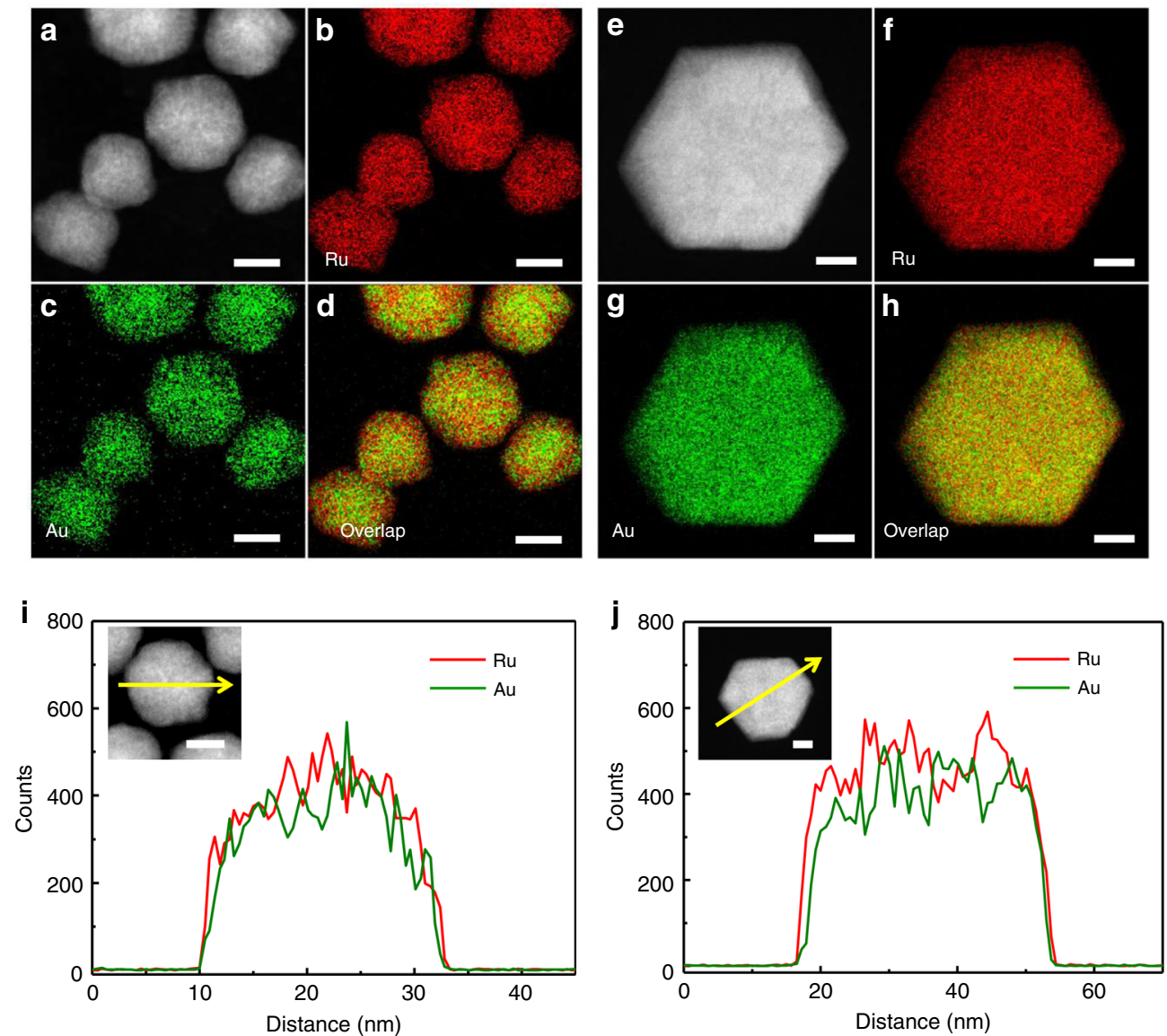

Fig. 1 EDX maps and line profiles of the synthesized $A A_{R u_{3}}$ NPs. a HAADF-STEM image of fcc-AuRu $u_{3}$ NPs. b Ru-L STEM-EDX map of (a). c Au-M STEM-EDX map of (a). d Overlay image of (b) and (c). e HAADF-STEM image of an hcp-AuRu 3 NP. $\mathbf{f}$ Ru-L STEM-EDX map of (e). $\mathbf{g}$ Au-M STEM-EDX

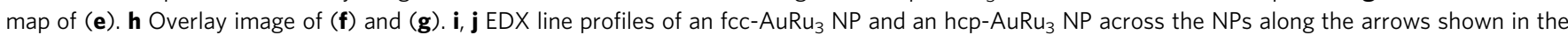
inset figure. Au and Ru are indicated as green and red lines, respectively. All of the scale bars shown in $(\mathbf{a}-\mathbf{j})$ are $10 \mathrm{~nm}$ 

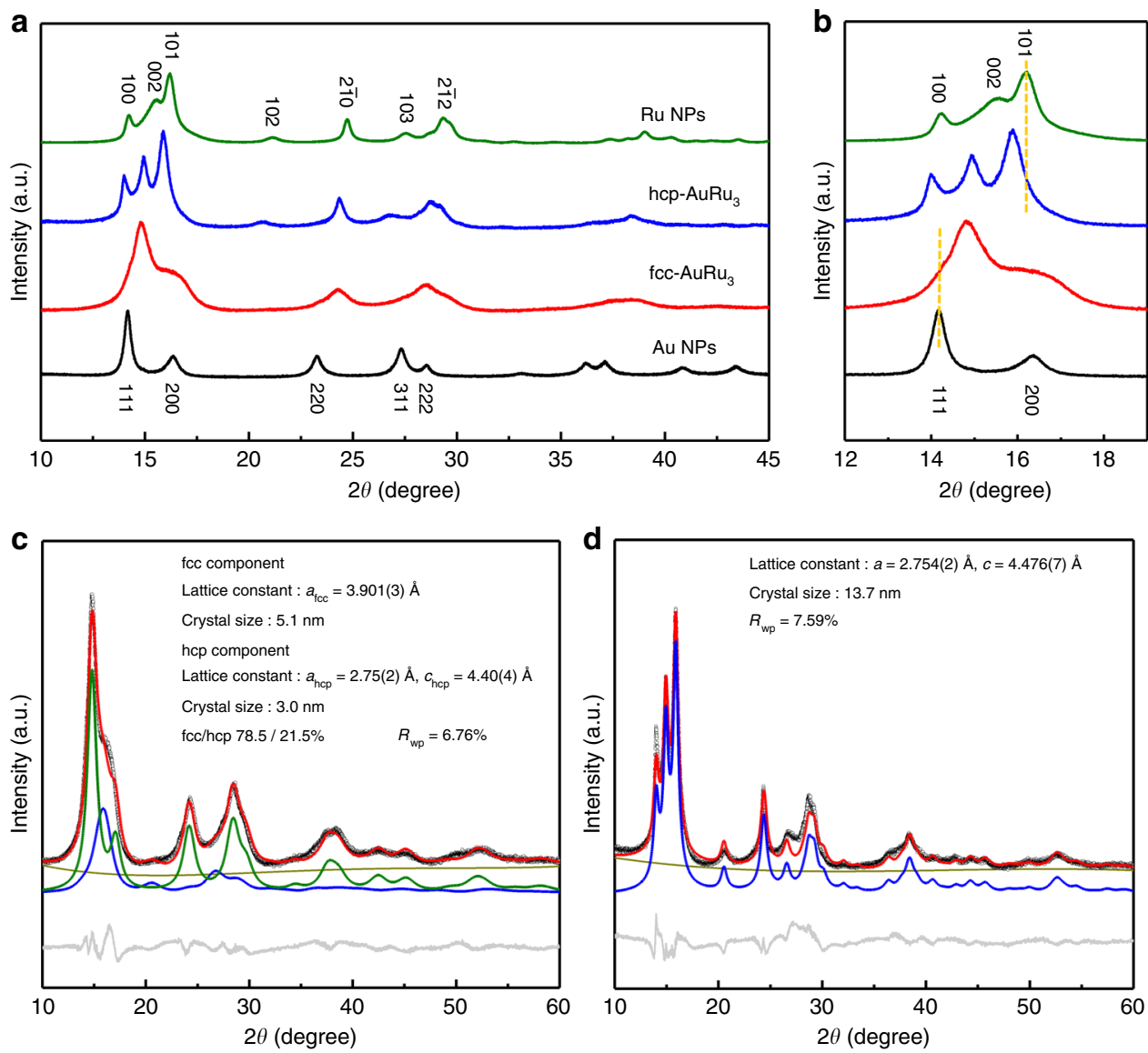

Fig. 2 Synchrotron XRD analysis of $A u R u_{3}$ NPs. a Synchrotron XRD patterns of $A u$, fcc-AuRu, hcp-AuRu $u_{3}$ and Ru NPs at $303 \mathrm{~K}, 2 \theta=10^{\circ}$ to $45^{\circ}$. $\mathbf{b}$ The close-up view of $2 \theta=12^{\circ}$ to $19^{\circ}$. The radiation wavelength was 0.57865 (1) $\AA$. c, d The Rietveld refinement for the fcc-AuRu $\mathrm{And}_{3} \mathrm{hcp}-\mathrm{AuRu}_{3} \mathrm{NPs}$. The diffraction patterns are shown as black circles. The calculated patterns are shown as red lines. The difference profile, the background profile and the fitting curves of the fcc and hcp components are shown as grey, dark yellow, green and blue lines, respectively

hydrogen tetrabromoaurate (III) hydrate $\left(\mathrm{HAuBr}_{4} \cdot \mathrm{nH}_{2} \mathrm{O}\right)$ and potassium pentachloronitrosylruthenate $(\mathrm{II})\left(\mathrm{K}_{2} \mathrm{Ru}(\mathrm{NO}) \mathrm{Cl}_{5}\right)^{12}$ were dissolved in diethylene glycol (DEG) with a 1:3 molar ratio. Then, the metal precursor solution was slowly dropped into an ethylene glycol (EG) solution containing polyvinylpyrrolidone (PVP) at $190^{\circ} \mathrm{C}$. The temperature of the solution was maintained at $190^{\circ} \mathrm{C}$ during the dropping process. The NPs were separated by centrifuging after cooling to room temperature, as denoted by the fcc-AuRu 3 NPs in the following section. In contrast, the synthesis of the hcp AuRu 3 alloy NPs was performed via a polyol reduction method in which $\mathrm{HAuBr}_{4} \cdot \mathrm{nH}_{2} \mathrm{O}$ and ruthenium (III) chloride hydrate $\left(\mathrm{RuCl}_{3} \cdot \mathrm{nH}_{2} \mathrm{O}\right)$ were used as metal precursors. DEG and PVP were used as a reductant and a protective agent, respectively. In addition, to prepare $\mathrm{AuRu}_{3}$ alloy NPs with an hcp structure, cetyltrimethylammonium bromide (CTAB) was added to adjust the reduction speed of the metal precursors. $\mathrm{HAuBr}_{4}$, $\mathrm{RuCl}_{3}$ and CTAB were dissolved in DEG in a 1:3:20 molar ratio. Then, the metal precursor solution was slowly dropped into a DEG solution containing PVP and CTAB at $215^{\circ} \mathrm{C}$. The NPs were separated by centrifuging after cooling to room temperature, as denoted by the hcp-AuRu $\mathrm{APs}_{3}$ in the following section.

Structural analysis. Transmission electron microscopy (TEM) images of the synthesized $\mathrm{AuRu}_{3}$ NPs were recorded using a Hitachi HT7700 TEM instrument at $100 \mathrm{kV}$ (Supplementary Fig. 1a, c). From the TEM images, the mean diameters of NPs were determined to be $15.8 \pm 2.9 \mathrm{~nm}$ for fcc- $\mathrm{AuRu}_{3} \mathrm{NPs}$ and 85.2 $\pm 6.6 \mathrm{~nm}$ for hcp- $\mathrm{AuRu}_{3} \mathrm{NPs}$. The mean diameters were estimated by averaging more than 500 particles (Supplementary Figs. 1b, d).

STEM-energy-dispersive X-ray (EDX) analyses were performed to obtain the direct evidence of the formation of a solid-solution structure. Figure $1 \mathrm{a}-\mathrm{d}$ and $\mathrm{e}-\mathrm{h}$ shows high-angle annular dark-field (HAADF)-STEM images, the corresponding $\mathrm{Ru}-\mathrm{L}$ and $\mathrm{Au}-\mathrm{M}$ STEM-EDX maps and overlays of $\mathrm{Au}$ and $\mathrm{Ru}$ maps of fcc-AuRu $\mathrm{And}_{3}$ and $\mathrm{hcp}-\mathrm{AuRu}_{3}$. In addition, lowmagnification EDX maps of the synthesized fcc- $\mathrm{AuRu}_{3}$ and hcp- $\mathrm{AuRu}_{3} \mathrm{NPs}$ are shown in Supplementary Figs. 2 and 3. These maps clearly show that $\mathrm{Au}$ and $\mathrm{Ru}$ atoms are randomly and homogeneously distributed in each NP of fcc- $\mathrm{AuRu}_{3}$ and hcp$\mathrm{AuRu}_{3}$, although these two elements cannot be mixed with each other, even in the liquid phase in the bulk state. In addition, from the EDX results, the ratios of $\mathrm{Au}$ to $\mathrm{Ru}$ in fcc- $\mathrm{AuRu}_{3}$ and hcp$\mathrm{AuRu}_{3}$ are 0.26:0.74 and 0.23:0.77, respectively, which are equal to the nominal ratio in the syntheses. The metal composition of the synthesized NPs was also analysed by using X-ray fluorescence spectroscopy (XRF). The ratios of Au to Ru in fcc$\mathrm{AuRu}_{3}$ and hcp-AuRu $\mathrm{Are}_{3}$ 0.25:0.75 and 0.24:0.76, respectively, which are consistent with the EDX results. We further characterized $\mathrm{AuRu}_{3} \mathrm{NPs}$ via the EDX line scanning analysis (Fig. 1i, j). The direction of the line scan is denoted by an arrow across the NP in Fig. 1i, j. These results also show that $\mathrm{Au}$ and $\mathrm{Ru}$ atoms are homogeneously distributed over the whole particles. These results indicate the formation of $\mathrm{AuRu}_{3}$ solid-solution alloy NPs. 

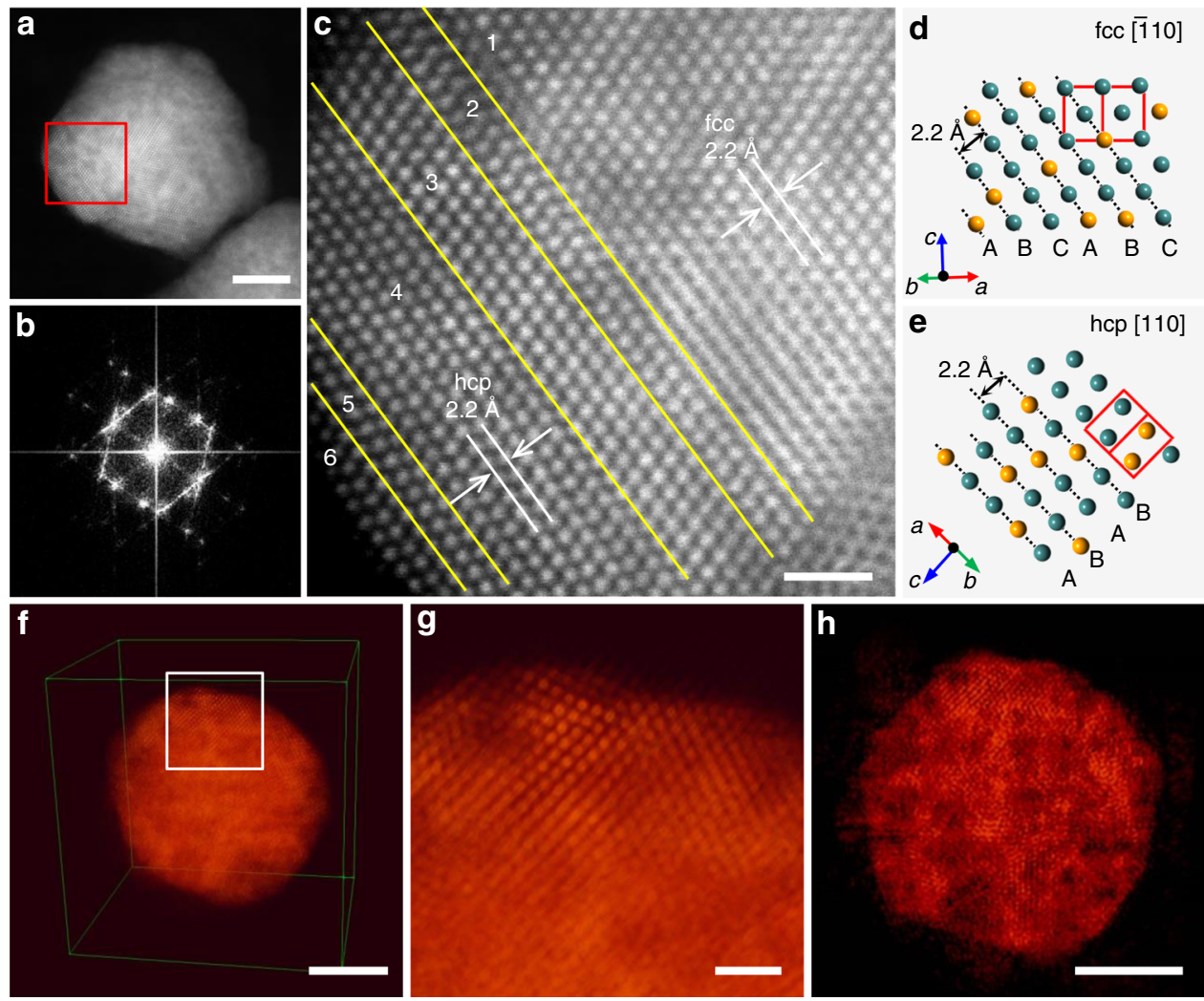

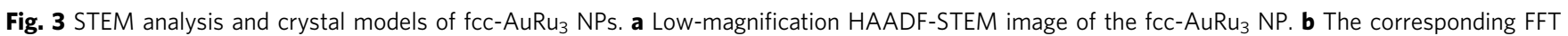
pattern of the NP in (a). c The atomic resolution HAADF-STEM image of the red square region in (a). $\mathbf{d}$ Model for the atomic arrangement of fcc structure viewed along the [110] direction. The unit cell is shown as the red frame. e Model for the atomic arrangement of the hcp structure viewed along the [110] direction. The unit cell is shown as the red frame. $\mathbf{f}$ The tomographic reconstruction of the fcc-AuRu $\mathrm{NP}_{3}$ by EST reconstruction algorithm. $\mathbf{g}$ Magnified image of the white square region in (f). h The $0.8 \AA$-thick slice image of the $3 \mathrm{D}$ reconstruction near the centre of the NP. The scale bars in (a), (c), (f), (g) and (h) are 5, 1, 5, 1 and $5 \mathrm{~nm}$

To investigate the crystal structures of the obtained $\mathrm{AuRu}_{3}$ solid-solution NPs, XRD measurements were carried out at 303 $\mathrm{K}$, at the beamline BL02B2, SPring- $8^{13}$. Figure 2a shows the XRD patterns of $\mathrm{Au}, \mathrm{fcc}-\mathrm{AuRu}_{3}, \mathrm{hcp}-\mathrm{AuRu}_{3}$ and $\mathrm{Ru}$ NPs. It is obvious that $\mathrm{Au}$ and $\mathrm{Ru}$ NPs show fcc and hcp diffraction patterns corresponding to their bulk metals. We found that the diffraction pattern of fcc- $\mathrm{AuRu}_{3} \mathrm{NPs}$ is completely different from that of hcp-AuRu $\mathrm{APs}_{3}$. The diffraction patterns of fcc-AuRu $\mathrm{An}_{3}$ and hcp$\mathrm{AuRu}_{3} \mathrm{NPs}$ were similar to those of $\mathrm{Au}$ and $\mathrm{Ru} \mathrm{NPs}$, respectively. However, the peak positions of alloy NPs clearly shifted from those of pure $\mathrm{Au}$ or $\mathrm{Ru} \mathrm{NPs}$ (Fig. 2b). This also indicates the formation of AuRu solid-solution alloy.

To clarify the crystal structures of the obtained NPs, the diffraction profiles were analysed by Rietveld refinement (Fig. 2c, d). The result calculated for fcc-AuRu $\mathrm{AP}_{3} \mathrm{NPs}$ revealed that the NPs consist of a major fcc phase $(78.5 \%)$ and a minor hcp phase $(21.5 \%)$. The lattice constant for the fcc component is 3.901(3) $\AA$, which is smaller than that of Au NPs $(a=4.077(3) \AA$, Supplementary Fig. 4), and the lattice constants of the hcp component are 2.75(2) and 4.40(4) $\AA$ for $a_{\mathrm{hcp}}$ and $c_{\mathrm{hcp}}$, which are larger than those of $\mathrm{Ru}$ NPs $(a=2.709(1) \AA, c=4.310(6) \AA$, Supplementary Fig. 5). The lattice parameter $a_{\mathrm{fcc}}$ in a fcc structure is approximately $\sqrt{ } 2 a_{\mathrm{hcp}}$ in an hcp structure because both the fcc and hcp structures are close-packed structures. Assuming that the lattice constant follows Vegard's law ${ }^{14}$, the $\mathrm{Au} / \mathrm{Ru}$ atomic ratios of the fcc and hcp phases are calculated to be 0.28:0.72 and 0.24:0.76, which are almost the same values as those found in the EDX analysis. Note that the metal composition of the fcc and hcp phases in fcc-AuRu $\mathrm{As}_{3}$ is equal and that the fcc phase ratio is greater than $75 \%$, even though there are two phases in the synthesized NPs. These results clarified that the $\mathrm{AuRu}_{3}$ solid-solution NPs with the fcc-phase-dominated crystal structure were successfully prepared.

In contrast, the diffraction profile of hcp-AuRu $\mathrm{APs}_{3}$ could be fitted by only an hcp component (Fig. 2d). The lattice constants of the hcp component are 2.754(2) $\AA$ for $a$ and 4.476(7) $\AA$ for $c$ which are larger than that of $\mathrm{Ru}$, and they are almost consistent with the lattice constants of the hcp component in fcc-AuRu alloy NPs. From Vegard's law, the Au/Ru atomic ratio of hcp$\mathrm{AuRu}_{3} \mathrm{NPs}$ is calculated to be $0.26: 0.74$, which is also in accordance with the EDX analysis result. These results confirmed the formation of the solid-solution hcp $\mathrm{AuRu}_{3}$ alloy NPs. Thus, we successfully showed the first example of selectively controlling the crystal structure of solid-solution alloy in an immiscible $\mathrm{Au}-\mathrm{Ru}$ system.

We further explored the crystal structure of fcc-AuRu $\mathrm{An}_{3}$ and hcp-AuRu $\mathrm{APs}_{3}$ using atomic resolution STEM. Figure 3a, b shows an HAADF-STEM image and a relevant fast Fourier transform (FFT) pattern obtained from the fcc-AuRu $\mathrm{Au}_{3}$ particle shown in Fig. 3a. These present a polycrystalline nature of the alloy NP, which is consistent with the fact that the crystal size calculated from the XRD pattern of fcc- $\mathrm{AuRu}_{3}$ is smaller than the average particle size observed from TEM. Figure $3 \mathrm{c}$ shows a clear atomic arrangement of the red area in Fig. 3a. The yellow lines indicate grain boundaries in the particle. The typical atomic arrangement of the fcc phase (ABCABC... stacking sequence of closed packed planes) was observed in the odd-numbered regions, whereas the typical atomic arrangement of the hcp phase 

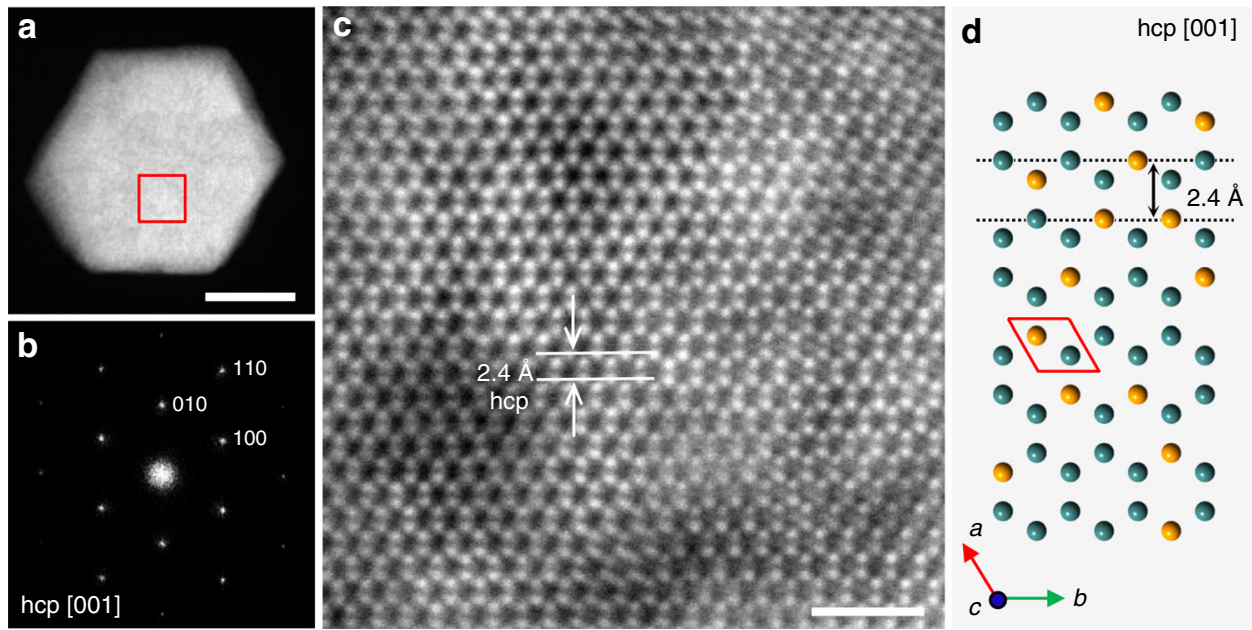

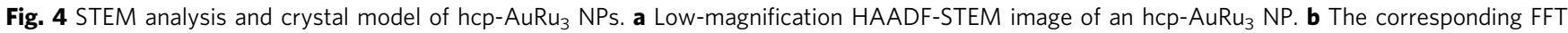
pattern of the NP in (a). c Atomic resolution HAADF-STEM image of the red square region in (a). d Model for the atomic arrangement of the hcp structure viewed along the [001] direction. The unit cell is shown as the red frame. The scale bars in (a) and (c) are 20 and $1 \mathrm{~nm}$

(ABAB...stacking sequence of closed packed planes) was observed in the even-numbered regions. The FFT patterns obtained from the selected areas in Fig. $3 \mathrm{c}$ also confirmed the fcc and hcp phases (Supplementary Fig. 6). The FFT pattern obtained from region A in Supplementary Fig. 6 fits the fcc crystal structure with a symmetry of $\mathrm{Fm} \overline{3} \mathrm{~m}$, as viewed along the [ $\left.\begin{array}{ll}\overline{1} & 10\end{array}\right]$ direction. The FFT pattern obtained from region $\mathrm{B}$ in Supplementary Fig. 6 fits the hcp crystal structure with a symmetry of $\mathrm{P}_{3} / \mathrm{mmc}$, as viewed along the [110] direction. For further comparison and confirmation, the atomic arrangements of the fcc structure along the [ $\left[\begin{array}{ll}1 & 10\end{array}\right]$ direction and the hcp structure along the [110] direction were simulated (Fig. 3d, e) and are consistent with the atomic arrangements obtained experimentally. In addition, the lattice spacings of both the fcc and hcp phases are observed to be $2.2 \AA$, which are consistent with the values given via the Rietveld refinement. However, as seen in Fig. 3c, most of the particles consist of the fcc phase, and hcp phases are observed as a fraction of grains between fcc phases like stacking faults.

To investigate the three-dimensional (3D) arrangement of atoms in fcc-AuRu 3 NPs, we performed atomic resolution electron tomography with an equally sloped tomography (EST) iterative reconstruction algorithm ${ }^{15-18}$ (Fig. $3 \mathrm{f}-\mathrm{h}$, Supplementary movies 1 and 2). In the tomographic reconstruction, some grains are observed inside the NP, thus showing the polycrystalline nature of the NP. In addition, the random distribution of both atoms over the whole NP is observed because brighter and darker atoms indicate $\mathrm{Au}$ and $\mathrm{Ru}$ atoms, respectively, which confirms the solid-solution structure of the NP. Inside the particle, some brighter and darker regions were observed in several atomic-scale regions and were attributed to $\mathrm{Au}$ - and $\mathrm{Ru}$-rich regions, respectively. These regions were randomly distributed in both the fcc and hcp grains in three dimensions, and they were smaller than the grain size. This may be derived from the originally immiscible nature of the $\mathrm{Au}-\mathrm{Ru}$ system.

The crystal structure of hcp-AuRu $\mathrm{APs}_{3} \mathrm{NP}$ was also investigated using an atomic resolution STEM. Figure $4 \mathrm{a}, \mathrm{b}$ shows an HAADF-STEM image and a relevant FFT pattern obtained from the particle shown in Fig. $4 \mathrm{a}$. In contrast to fcc-AuRu $\mathrm{AP}_{3} \mathrm{NP}$, the FFT pattern in Fig. 4b fits a pure hcp crystal structure with a symmetry of $\mathrm{P}_{3} / \mathrm{mmc}$, viewed along the [001] direction. This presents a single crystalline nature of the alloy NP. Figure $4 \mathrm{c}$ shows a clear atomic arrangement of a typical hcp structure viewed along the [001] direction, which is consistent with the simulated model drawn in Fig. 4d. In addition, the lattice spacing of the (100) plane is observed as $2.4 \AA$, which is consistent with the Rietveld refinement result. Moreover, some bright and dark areas are observed in several atomic-scale regions as well as in the fcc- $\mathrm{AuRu}_{3} \mathrm{NP}$, while Fig. 4c shows a single hcp arrangement.

Mechanism of selective control of the crystal structure. We hypothesized that the selective control of crystal structure in solid-solution alloy NPs can be achieved by finely tuning the reduction speeds of metal precursors with a very small difference. In general, simultaneous reduction of metal precursors is necessary for the formation of solid-solution alloy NPs ${ }^{19-22}$. Otherwise, phase-separated NPs, such as core-shell or segregated types, will be obtained ${ }^{23}$. However, if the reduction occurs completely simultaneously, it may be difficult to control the crystal structure of the solid-solution alloy. We believe that a very small difference in reduction speeds of precursors is needed for the phase control. The crystal structure of binary alloy NPs could be governed by the structure of nuclei made of one of the constituent metal ions, which starts to be reduced slightly earlier than the other during the alloy formation process. Thus, in the target system, if the reduction of $\mathrm{Au}$ precursor starts earlier than that of $\mathrm{Ru}$ precursor, alloy NPs would adopt a fcc structure since Au naturally forms a fcc structure, as shown in Fig. 5a. While, if the reduction of the $\mathrm{Ru}$ precursor starts earlier than that of the Au precursor, the alloy NPs would form an hcp structure because $\mathrm{Ru}$ favours an hcp structure.

To prove this hypothesis, the reduction process of $\mathrm{Au}$ and $\mathrm{Ru}$ precursors was investigated using UV-vis spectroscopy and cyclic voltammetry $(\mathrm{CV})$. To compare the reduction speed of the metal precursors during the formation of alloy NPs, we reduced each precursor under the same conditions used for the syntheses of alloy NPs and monitored the colour change of the solutions that accompanied the reduction of precursors by using UV-vis spectroscopy (Fig. 5b-e).

The fcc AuRu solid-solution alloy NPs were obtained by the reduction of $\mathrm{HAuBr}_{4}$ and $\mathrm{K}_{2} \mathrm{Ru}(\mathrm{NO}) \mathrm{Cl}_{5}$. In the spectra of solution (i) $\left(\mathrm{HAuBr}_{4} \cdot \mathrm{nH}_{2} \mathrm{O}\right.$ in $\left.\mathrm{EG}\right)$, as shown in Fig. $5 \mathrm{~b}$, a peak of $\left[\mathrm{AuBr}_{4}\right]^{-}$at approximately $400 \mathrm{~nm}$, which was assigned to ligandto-metal charge transfer ${ }^{24-26}$, disappeared in $10 \mathrm{~s}$. A peak at approximately $535 \mathrm{~nm}$, which was attributed to typical surface plasmon absorption of $\mathrm{Au} \mathrm{NPs}{ }^{27-29}$, appeared after $10 \mathrm{~s}$, and the intensity of the plasmon peak was saturated at $90 \mathrm{~s}$. In addition, in 
a
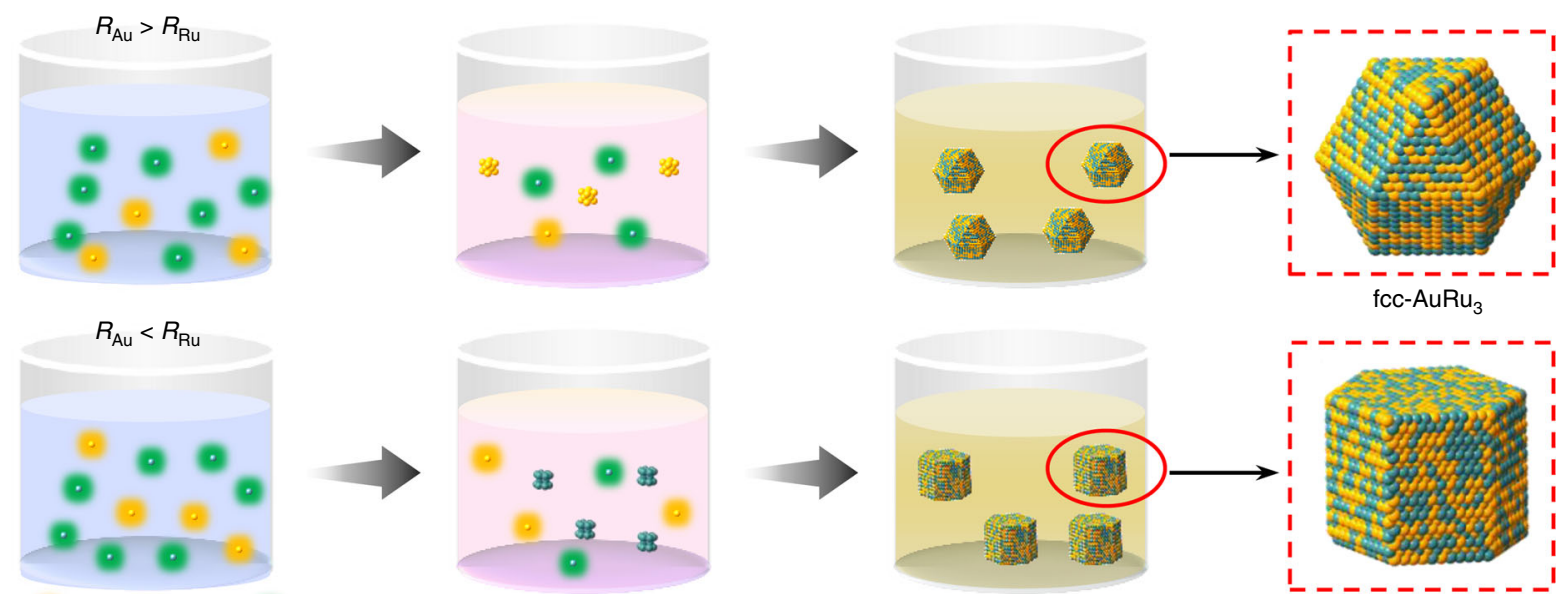

Au ion

Ru ion

Au crystal nucleus

I Ru crystal nucleus
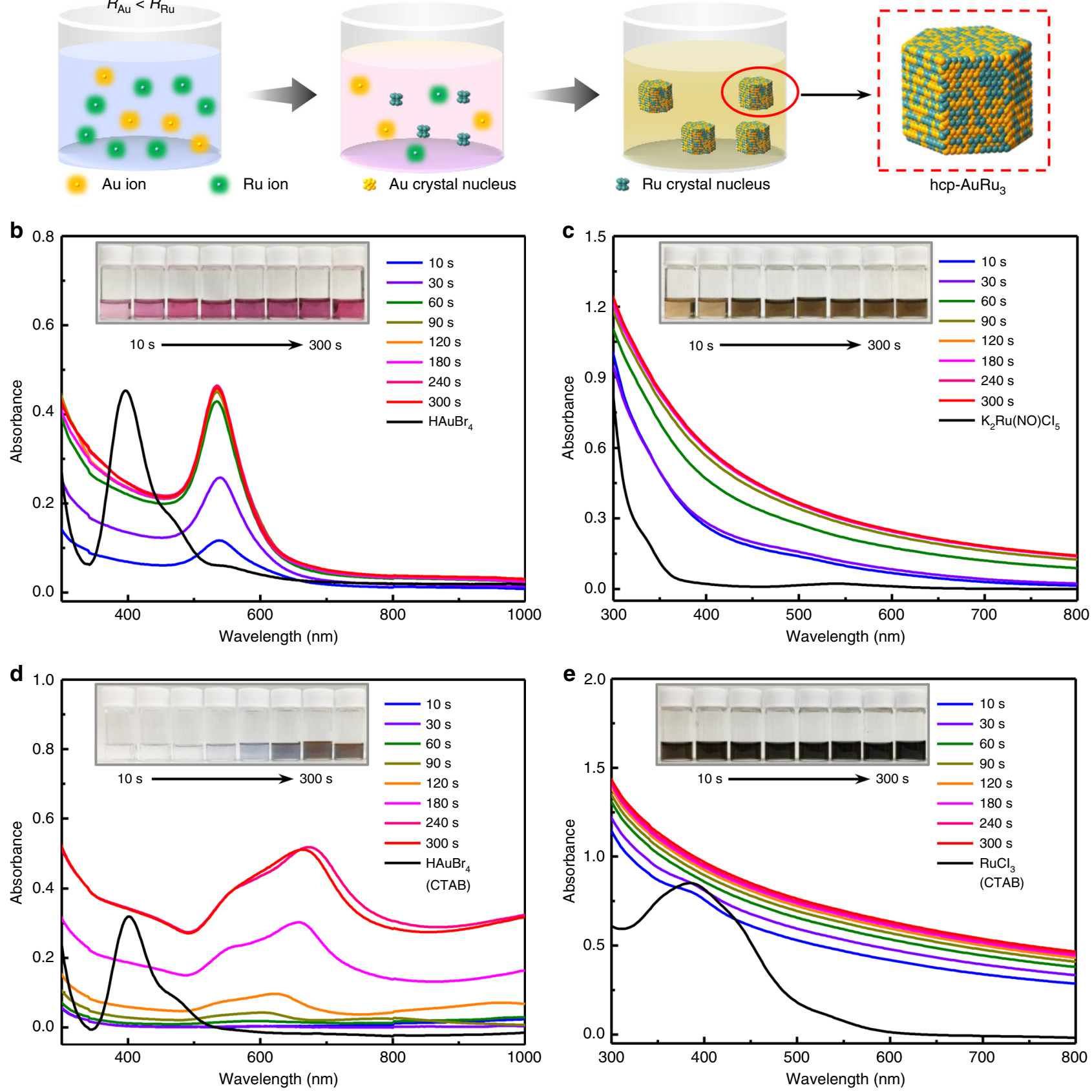

Fig. 5 Investigation of precursors' reduction speeds. a Schematic illustration of the formation process of AuRu $\mathrm{u}_{3}$ alloy NPs with fcc and hcp crystal structures. ( $R_{\mathrm{Au}}$ and $R_{\mathrm{Ru}}$ are the reduction speed of the $\mathrm{Au}$ and Ru precursors, respectively.) $\mathbf{b}, \mathbf{c}$ The change in $U V$-vis spectra of $\mathbf{b} \mathrm{HAuBr}{ }_{4}$ and $\mathbf{c} \mathrm{K}_{2} \mathrm{Ru}$ $(\mathrm{NO}) \mathrm{Cl}_{5}$ in $\mathrm{EG}$ with reaction time. $\mathbf{d}$, e The change in UV-vis spectra of $\mathbf{d} \mathrm{HAuBr}_{4}$ and $\mathbf{e} \mathrm{RuCl}_{3}$ with CTAB in DEG with reaction time. The inset pictures in (b), (c), (d) and (e) are the samples which were taken at different times 
the spectra of solution (ii) $\left(\mathrm{K}_{2} \mathrm{Ru}(\mathrm{NO}) \mathrm{Cl}_{5}\right.$ in $\left.\mathrm{EG}\right)$ (Fig. $\left.5 \mathrm{c}\right)$, a broad peak of $\left[\mathrm{Ru}(\mathrm{NO}) \mathrm{Cl}_{5}\right]^{2-}$ at approximately $545 \mathrm{~nm}$ disappeared, and another very broad peak at approximately $500 \mathrm{~nm}$ appeared after $10 \mathrm{~s}$, which was derived from the $\mathrm{Ru}^{2+} / \mathrm{NO}^{+}$ precursor $^{30}$. This broad peak gradually disappeared in $60 \mathrm{~s}$, and the colour of solution turned black, thus implying the formation of Ru NPs at the same time. The spectra did not change after $90 \mathrm{~s}$ and are similar to that of the Ru NP solution (Supplementary Fig. 8). By comparing these results, it is suggested that the Au precursor $\left(\mathrm{HAuBr}_{4}\right)$ is reduced slightly faster than the $\mathrm{Ru}$ precursor $\left(\mathrm{K}_{2} \mathrm{Ru}(\mathrm{NO}) \mathrm{Cl}_{5}\right)$ under the same conditions used for the synthesis of fcc $\mathrm{AuRu}_{3}$ NPs.

In contrast, hep AuRu solid-solution alloy NPs were synthesized via the reduction of $\mathrm{HAuBr}_{4}$ and $\mathrm{RuCl}_{3}$ with CTAB. In the spectra of solution (iii) $\left(\mathrm{HAuBr}_{4} \cdot \mathrm{nH}_{2} \mathrm{O}\right.$ with $\mathrm{CTAB}$ in DEG) (Fig. 5d), the peak of $\left[\mathrm{AuBr}_{4}\right]^{-}$at approximately $400 \mathrm{~nm}$ disappeared in $10 \mathrm{~s}$ and became colourless. Then, absorption peaks at approximately $500-700 \mathrm{~nm}$, which were attributed to the surface plasmon absorption of Au NPs, appeared after $90 \mathrm{~s}$, and a redshift of the peak at approximately $600 \mathrm{~nm}$ was observed with increasing particle size ${ }^{31-33}$ (Supplementary Fig. 9). In addition, in the spectra of solution (iv) $\left(\mathrm{RuCl}_{3} \cdot \mathrm{nH}_{2} \mathrm{O}\right.$ with CTAB in DEG) (Fig. 5e), a broad peak of $\mathrm{RuCl}_{3}$ at approximately $400 \mathrm{~nm}^{34-36}$ gradually disappeared in $60 \mathrm{~s}$. No peak was observed after $60 \mathrm{~s}$, and the spectra were similar to that of the Ru NP solution (Supplementary Fig. 8). By comparing these results, it is suggested that the $\mathrm{Ru}$ precursor $\left(\mathrm{RuCl}_{3}\right)$ is reduced slightly faster than the $\mathrm{Au}$ precursor $\left(\mathrm{HAuBr}_{4}\right)$ under the same conditions used for the synthesis of hcp $\mathrm{AuRu}_{3}$ NPs.

In addition, $\mathrm{CTAB}$ plays a very important role in tuning the reduction speed of the Au precursor because the hcp alloy NPs cannot be obtained without CTAB (Supplementary Figs. 10 and 11). Therefore, we compared the difference between the UV-vis spectra of Au precursors with and without CTAB (Fig. 5d and Supplementary Fig. 12). Without CTAB, Au plasmon absorption peaks were observed at $10 \mathrm{~s}$, while those peaks were observed at $90 \mathrm{~s}$ in Fig. 5d. We further investigated the effect of $\mathrm{CTAB}$ on the reduction speed of Au by CV (Supplementary Fig. 13). The reduction potential of $\left[\mathrm{AuBr}_{4}\right]^{-}$without $\mathrm{CTAB}$ was measured to be $0.856 \mathrm{~V}$, which is almost in accordance with the value given in the literature ${ }^{37-39}$. However, the reduction potential of $\left[\mathrm{AuBr}_{4}\right]^{-}$with $\mathrm{CTAB}$ was $0.768 \mathrm{~V}$, which was lower than that of $\left[\mathrm{AuBr}_{4}\right]^{-}$without CTAB. From these results, it is confirmed that the reduction speed of $\left[\mathrm{AuBr}_{4}\right]^{-}$can be tuned by CTAB. This is probably caused by a complex formation between the $\mathrm{Au}$ precursor and $\mathrm{CTAB}^{40,41}$. These investigations proved our hypothesis that fine-tuning the reduction speed of metal precursors with a very small difference can realize the selective control of the crystal structure in solid-solution alloy NPs.

\section{Discussion}

Recently, the selective control of the crystal structure in monometallic NPs has received significant attention as an attractive strategy to control their properties. The selective control of the crystal structure in bimetallic NPs at an arbitrary compositional ratio is highly desired to create more effective materials. In this study, we proposed an approach for the selective control of the crystal structure in solid-solution alloys at the same metal composition by finely tuning the reduction speed of the metal precursors with a very small difference, and we demonstrated an example of selective control by synthesizing fcc and hcp $\mathrm{AuRu}_{3}$ alloy NPs. Fine-tuning the reduction speed of the metal precursors was achieved by selecting the appropriate precursors and using CTAB. The combination of $\mathrm{HAuBr}_{4}$ and $\mathrm{K}_{2} \mathrm{Ru}(\mathrm{NO}) \mathrm{Cl}_{5}$ in which the Au precursor starts to be reduced slightly earlier provided a fcc structure, while the combination of $\mathrm{HAuBr}_{4}$ and $\mathrm{RuCl}_{3}$ with CTAB, in which the $\mathrm{Ru}$ precursor begins to be reduced slightly earlier, provided an hcp structure.

In general, the properties of alloy NPs have been controlled by the size, morphology, constituent elements and compositional ratio. In this work, we introduced a basic material design degree of freedom "crystal structure" to create innovative chemical and physical properties for alloy NPs. Furthermore, this concept can provide a method for controlling the crystal structure of not only $\mathrm{Au}-\mathrm{Ru}$ system but also other alloy systems consisting of several elements that adopt different structures for the development of innovative electronic, optical, magnetic and catalytic materials.

\section{Methods}

Preparation of fcc-AuRu $\mathbf{u}_{\mathbf{3}}$ NPs. The fcc alloy NPs were prepared using a polyol reduction method. First, PVP (444 mg, molecular weight $\approx 40000$, Wako) was dissolved in EG (100 mL, Wako). Then, the solution was heated to $190{ }^{\circ} \mathrm{C}$ with magnetic stirring. Following this, the precursor solution, which was prepared by dissolving hydrogen tetrabromoaurate (III) hydrate $\left(\mathrm{HAuBr}_{4} \bullet \mathrm{nH}_{2} \mathrm{O}, 15.2 \mathrm{mg}\right.$, Alfa Aesar) and potassium pentachloronitrosylruthenate (II) $\left(\mathrm{K}_{2} \mathrm{Ru}(\mathrm{NO}) \mathrm{Cl}_{5}, 29.0 \mathrm{mg}\right.$, Aldrich) in $10 \mathrm{~mL}$ of DEG, was dropped into the hot EG solution at a rate of 1.5 $\mathrm{mL} \min ^{-1}$ using a syringe infusion pump (KDS 200). The temperature of the solution was maintained at approximately $190^{\circ} \mathrm{C}$ during the dropping process. After the dropping process, the solution was kept at the same temperature for another $10 \mathrm{~min}$. Then, the NPs were separated via centrifuging after cooling to room temperature.

Preparation of hcp-AuRu $\mathbf{3}$ NPs. PVP (444 mg) and CTAB (364.4 mg, Tokyo Chemical Industry Co., Ltd) were dissolved in $100 \mathrm{~mL}$ of DEG, and the solution was heated to $215^{\circ} \mathrm{C}$ with magnetic stirring. Then, the precursor solution, which was prepared by dissolving $\mathrm{HAuBr}_{4} \bullet \mathrm{nH}_{2} \mathrm{O}(15.2 \mathrm{mg}$, Alfa Aesar), ruthenium (III) chloride hydrate $\left(\mathrm{RuCl}_{3} \cdot \mathrm{nH}_{2} \mathrm{O}, 19.6 \mathrm{mg}\right.$, Wako) and CTAB $(182.2 \mathrm{mg})$ in $10 \mathrm{~mL}$ of DEG, was dropped into the hot DEG solution with a speed of $1.5 \mathrm{~mL} \mathrm{~min}{ }^{-1}$. The temperature of the solution was maintained at $215^{\circ} \mathrm{C}$ during the dropping process After the dropping process, the solution was kept at the same temperature for another $10 \mathrm{~min}$. Then, the NPs were separated by centrifuging after cooling to room temperature.

Characterization. Synchrotron XRD patterns were measured at the BL02B2 beamline, SPring- 8 at room temperature. The radiation wavelength was $0.5786 \AA$ TEM images were acquired using a Hitachi HT7700 operated at $100 \mathrm{kV}$. The HAADF-STEM images and EDX spectra were captured using a JEOL JEMARM200CF STEM instrument operated at $200 \mathrm{kV}$. XRF was measured using a Rigaku ZSX Primus IV. Absorption spectra of the solutions were recorded using a JascoV-570 UV-vis spectrophotometer.

Rietveld refinement. The Rietveld refinements were performed by using TOPAS3 software developed by Bruker AXS GmbH. The full width at half maximum of silicon (Standard Reference Material 640c) 111 diffraction peak is about $0.015^{\circ}$ at $2 \theta=10^{\circ}$ which is an instrumental resolution function of the diffractometer. All XRD patterns were refined in $2 \theta$ range from 10 to $60^{\circ}$ with $0.006^{\circ}$ per step identical to the resolution of the beamline BL02B2, SPring- 8 . The XRD pattern of fcc-AuRu 3 was well fitted by two components with space groups of $\mathrm{Fm} \overline{3}$ $\mathrm{m}$ and $\mathrm{P}_{3} / \mathrm{mmc}$. For hcp-AuRu $\mathrm{H}_{3}$, the pattern was fitted by one component of $\mathrm{P}_{3} / \mathrm{mmc}$.

HAADF-STEM tomography. The tilt series of HAADF-STEM images for the individual fcc- $\mathrm{AuRu}_{3} \mathrm{NP}$ was acquired at the angle range from $-55.6^{\circ}$ to $65.6^{\circ}$ using JEM-ARM200F operated at $120 \mathrm{kV}$ (Supplementary Fig. 7). The image size was $1024 \times 1024$ pixels at a pixel size of $0.20 \times 0.20 \AA^{2}$. To reduce electron beam damage, 10 images were acquired using a small pixel time of $1 \mu \mathrm{sx}^{-1}$ and a probe current of $12 \mathrm{pA}$ at each angle step. The image series at each angle step were averaged, and the image size was reduced to $512 \times 512$ pixels, after image alignment and affine-transforms to correct image distortion due to specimen drifts. The intensity of the averaged tilt image series was normalized using the intensity of a carbon film part to correct the intensity variation due to the electron probe current changes. The background of each projection was removed after alignment using the cross-correlation method ${ }^{42}$. The EST reconstruction algorithm ${ }^{15-17}$ developed by the UCLA group and their codes were used for 3D tomographic reconstruction. In EST reconstruction, Fourier-space-based iterative reconstructions were performed with real-space constrains to minimize the difference between observed images and the reconstruction projections using a pseudo polar fast Fourier transform (PPFFT) and the inverse PPFFT with oversampling ${ }^{15-17}$. The detailed scheme of the EST reconstruction algorism was described in the supporting information of ref. ${ }^{15}$. In this study, the 500 times iteration was carried out to minimize the R-factor and two 
times oversampling PPFFT were used. The reconstructed data were visualized via the Visualizer Kai post processing software (Systems In Frontiers Inc.).

Reduction speed measurements. To compare the reduction speed of metal precursors during the formation of alloy NPs, each precursor was reduced under the same conditions used for the syntheses of alloy NPs and detected the colour change of the solutions accompanying the reduction of precursors via the UV-vis spectroscopy. The measurements for each metal precursor were repeated at least twice. In a standard procedure, the metal precursor used for the formation of fcc$\mathrm{AuRu}_{3} \mathrm{NPs}, \mathrm{K}_{2} \mathrm{Ru}(\mathrm{NO}) \mathrm{Cl}_{5}(0.075 \mathrm{mmol})$ or $\mathrm{HAuBr}_{4}(0.025 \mathrm{mmol})$, was first dissolved in $10 \mathrm{~mL}$ of EG. Then, $100 \mathrm{~mL}$ of the EG solvent was heated to $195^{\circ} \mathrm{C}$ with stirring. Then, the precursor solvent was quickly injected into the hot EG solvent and was heated at a temperature of approximately $190^{\circ} \mathrm{C}$. During the heating process, $1.5 \mathrm{~mL}$ aliquots were sampled from the reaction solution using a glass pipet and immediately immersed into an ice bath to quickly quench the reduction reaction. The samples were taken at 10,30,60,90,120, 180,240 and $300 \mathrm{~s}$ and diluted to $3 \mathrm{~mL}$ by using EG. The absorption spectra of samples were measured using a UV-vis spectrophotometer. The same procedure was performed to investigate the reduction process of metal precursors used in the formation of hcp$\mathrm{AuRu}_{3}$ NPs. The differences were that DEG was used as a solvent instead of EG and CTAB $(1.5 \mathrm{mmol})$ was added to the solvent. The temperature was kept at approximately $215^{\circ} \mathrm{C}$.

Electrochemical measurements. The concentrations of $\mathrm{HAuBr}_{4}$ and $\mathrm{CTAB}$ were $0.5 \mathrm{mmol} \mathrm{L}^{-1}$ and $10 \mathrm{mmol} \mathrm{L}^{-1}$. A CHI $760 \mathrm{E}$ electrochemical analyser $(\mathrm{CH}$ Instruments) was used for collecting the electrochemical data. All of the experiments were performed at room temperature using a conventional three-electrode system with a $3.0 \mathrm{~mm}$ diameter glassy carbon disk working electrode, a platinum wire auxiliary electrode and a $\mathrm{Ag} / \mathrm{AgCl}(3 \mathrm{M} \mathrm{NaCl})$ reference electrode. All of the electrodes were purchased from ALS Co., Ltd. The working electrode surface was polished with $1 \mu \mathrm{M}$ diamond and $0.05 \mu \mathrm{M}$ alumina solutions (ALS Co., Ltd) before the tests. The measurements were carried out in an Ar-saturated $0.05 \mathrm{M} \mathrm{H}_{2} \mathrm{SO}_{4}$ aqueous solution. During the measurement, the atmosphere above the solution was kept inert with a constant flow of Ar. The cyclic voltammograms were recorded at a scan rate of $0.02 \mathrm{~V} \mathrm{~s}^{-1}$ and measured over the potential range of +0.7 to $-0.1 \mathrm{~V}$ (vs. $\mathrm{Ag} / \mathrm{AgCl}$ ).

Data availability. The authors declare that the data supporting the findings of this study are available within the paper and its supplementary information files.

Received: 16 October 2017 Accepted: 9 January 2018

Published online: 06 February 2018

\section{References}

1. Cox, P. A. The Electronic Structure and Chemistry of Solids (Oxford University Press, New York, 1987).

2. Kittel, C. Introduction to Solid State Physics (John Wiley \& Sons, Inc., New York, 2005).

3. Massalski, T. B., Okamoto, H., Subramanian, P. R. \& Kacprzak, L. Binary Alloy Phase Diagrams (ASM International, Materials Park, 1996).

4. Qadri, S. B. et al. Structural and magnetic properties of nanocrystalline RuCo alloys. Appl. Phys. Lett. 91, 214101 (2007).

5. Kusada, K. et al. Solid solution alloy nanoparticles of immiscible Pd and Ru elements neighboring on $\mathrm{Rh}$ : changeover of the thermodynamic behavior for hydrogen storage and enhanced CO-oxidizing ability. J. Am. Chem. Soc. 136, 1864-1871 (2014).

6. Fan, Z. \& Zhang, H. Crystal phase-controlled synthesis, properties and applications of noble metal nanomaterials. Chem. Soc. Rev. 45, 63-82 (2016).

7. Kusada, K. \& Kitagawa, H. A route for phase control in metal nanoparticles: a potential strategy to create advanced materials. Adv. Mater. 28, 1129-1142 (2016).

8. Huang, X. et al. Synthesis of hexagonal close-packed gold nanostructures. Nat. Commun. 2, 292 (2011).

9. Fan, Z. et al. Surface modification-induced phase transformation of hexagonal close-packed gold square sheets. Nat. Commun. 6, 6571 (2015).

10. Kusada, K. et al. Discovery of face-centered-cubic ruthenium nanoparticles: facile size-controlled synthesis using the chemical reduction method. J. Am. Chem. Soc. 135, 5493-5496 (2013).

11. Okamoto, H. \& Massalski, T. B. The Au-Ru (Gold-Ruthenium) system. J. Phase Equilib. 5, 388-390 (1984).

12. Veal, J. T. \& Hodgson, D. J. The crystal and molecular structure of potassium pentaehloronitrosylruthenate(II), $\mathrm{K}_{2}\left[\mathrm{Ru}\left(\mathrm{NO} \mathrm{CI}_{5}\right]\right.$. Acta Cryst. B28, 3525-3529 (1972).
13. Kawaguchi, S. et al. High-throughput powder diffraction measurement system consisting of multiple MYTHEN detectors at beamline BL02B2 of SPring-8. Rev. Sci. Instrum. 88, 085111 (2017).

14. Denton, A. R. \& Ashcroft, N. W. Vegard's law. Phy. Rev. A 43, 3161-3164 (1991).

15. Scott, M. C. et al. Electron tomography at 2.4-ångstrom resolution. Nature 483, 444-447 (2012).

16. Chen, C. C. et al. Three-dimensional imaging of dislocations in a nanoparticle at atomic resolution. Nature 496, 74-77 (2013).

17. $\mathrm{Xu}, \mathrm{R}$. et al. Three-dimensional coordinates of individual atoms in materials revealed by electron tomography. Nat. Mater. 14, 1099-1103 (2015).

18. Miao, J., Ercius, P. \& Billinge, S. J. Atomic electron tomography: 3D structures without crystals. Science 353, aaf2157 (2016).

19. Kusada, K., Yamauchi, M., Kobayashi, H., Kitagawa, H. \& Kubota, Y. Hydrogen-storage properties of solid-solution alloys of immiscible neighboring elements with Pd. J. Am. Chem. Soc. 132, 15896-15898 (2010).

20. Wu, D., Cao, M., Shen, M. \& Cao, R. Sub-5 nm Pd-Ru nanoparticle alloys as efficient catalysts for formic acid electrooxidation. ChemCatChem 6, 1731-1736 (2014)

21. Komatsu, T. et al. First-principles calculation, synthesis, and catalytic properties of $\mathrm{Rh}-\mathrm{Cu}$ alloy nanoparticles. Chem. Eur. J. 23, 57-60 (2017)

22. Huang, B. et al. Solid-solution alloying of immiscible $\mathrm{Ru}$ and $\mathrm{Cu}$ with enhanced CO oxidation activity. J. Am. Chem. Soc. 139, 4643-4646 (2017).

23. Wu, D., Zheng, Z., Gao, S., Cao, M. \& Cao, R. Mixed-phase PdRu bimetallic structures with high activity and stability for formic acid electrooxidation. Phys. Chem. Chem. Phys. 14, 8051-8057 (2012).

24. Gangopadhayay, A. K. \& Chakravorty, A. Charge transfer spectra of some gold(III) complexes. J. Chem. Phys. 35, 2206-2209 (1961).

25. Isci, H. \& Mason, W. R. Ligand-to-metal charge-transfer spectra of tetrahaloaurate(III) and trans-dicyanodihaloaurate(III) ions. Inorg. Chem. 22, 2266-2272 (1983)

26. Eustis, S. \& El-Sayed, M. A. Molecular mechanism of the photochemical generation of gold nanoparticles in ethylene glycol: support for the disproportionation mechanism. J. Phys. Chem. B 110, 14014-14019 (2006).

27. Grunwaldt, J. D., Kiener, C., Wögerbauer, C. \& Baiker, A. Preparation of supported gold catalysts for low-temperature CO oxidation via "sizecontrolled" gold colloids. J. Catal. 181, 223-232 (1999).

28. Pérez-Juste, J., Liz-Marzán, L. M., Carnie, S., Chan, D. Y. C. \& Mulvaney, P. Electric-field-directed growth of gold nanorods in aqueous surfactant solutions. Adv. Funct. Mater. 14, 571-579 (2004).

29. Ghosh, S. K. \& Pal, T. Interparticle coupling effect on the surface plasmon resonance of gold nanoparticles: from theory to applications. Chem. Rev. 107, 4797-4862 (2007).

30. Lahiri, G. K. \& Kaim, W. Electronic structure alternatives in nitrosylruthenium complexes. Dalton Trans. 39, 4471-4478 (2010).

31. Link, S. \& El-Sayed, M. A. Size and temperature dependence of the plasmon absorption of colloidal gold nanoparticles. J. Phys. Chem. B 103, 4212-4217 (1999).

32. Zhong, Z., Patskovskyy, S., Bouvrette, P., Luong, J. H. T. \& Gedanken, A. The surface chemistry of Au colloids and their interactions with functional amino acids. J. Phys. Chem. B 108, 4046-4052 (2004).

33. Haiss, W., Thanh, N. T. K., Aveyard, J. \& Fernig, D. J. Determination of size and concentration of gold nanoparticles from UV-Vis spectra. Anal. Chem. 79, 4215-4221 (2007)

34. Yan, X. P., Liu, H. F. \& Liew, K. Y. Size control of polymer-stabilized ruthenium nanoparticles by polyol reduction. J. Mater. Chem. 11, 3387-3391 (2001).

35. Harpeness, R. et al. Controlling the agglomeration of anisotropic Ru nanoparticles by the microwave-polyol process. J. Colloid Interface Sci. 287, 678-684 (2005).

36. Erdoğan, H., Metin, Ö. \& Özkar, S. Hydrogen generation from the methanolysis of ammonia borane catalyzed by in situ generated, polymer stabilized ruthenium(0) nanoclusters. Catal. Today 170, 93-98 (2011).

37. Evans, D. H. \& Lingane, J. J. Standard potentials of the couples involving $\mathrm{AuBr}_{4}{ }^{-}, \mathrm{AuBr}_{2}{ }^{-}$and $\mathrm{Au}$ in bromide media. J. Electroanal. Chem. 6, 1-10 (1963).

38. Lide, D. R. Handbook of Chemistry and Physics (CRC Press: Boca Raton, 1989).

39. Grunder, Y., Ho, H. L., Mosselmans, J. F., Schroeder, S. L. \& Dryfe, R. A. Inhibited and enhanced nucleation of gold nanoparticles at the waterl1,2dichloroethane interface. Phys. Chem. Chem. Phys. 13, 15681-15689 (2011).

40. Torigoe, K. \& Esumi, K. Preparation of colloidal gold by photoreduction of $\mathrm{AuCl}_{4}{ }^{-}$-cationic surfactant complexes. Langmuir 8, 59-63 (1992).

41. Nikoobakht, B. \& El-Sayed, M. A. Preparation and growth mechanism of gold nanorods (NRs) using seed-mediated growth method. Chem. Mater. 15, 1957-1962 (2003). 
42. Haberfehlner, G. et al. Formation of bimetallic clusters in superfluid helium nanodroplets analysed by atomic resolution electron tomography. Nat. Commun. 6, 8779 (2015).

\section{Acknowledgements}

This research was supported by the ACCEL program, Japan Science and Technology Agency (JST), JPMJAC1501. STEM observations were performed as part of a program conducted by the Advanced Characterization Nanotechnology Platform sponsored by the MEXT of the Japanese Government. Synchrotron XRD measurements were carried out at SPring-8 under proposal No. 2014B1382, 2015A1586 and 2016A1483. The activities of the INAMORI Frontier Research Center, Kyushu University are supported by KYOCERA Corporation.

\section{Author contributions}

K.K. and H.K. conceived and designed the research. Q.Z. performed the experiments. T.Y., T. T. and S.M. performed the HAADF-STEM and EDX analyses. S.K. and Y.K. contributed to the synchrotron XRD measurements. Q.Z. and D.W. performed electrochemical experiments. Q.Z., K.K. and H.K. analysed and discussed the experimental results and wrote the manuscript. All of the authors discussed and commented on the paper.

\section{Additional information}

Supplementary Information accompanies this paper at https://doi.org/10.1038/s41467018-02933-6.
Competing interests: The authors declare no competing financial interests.

Reprints and permission information is available online at http://npg.nature.com/ reprintsandpermissions/

Publisher's note: Springer Nature remains neutral with regard to jurisdictional claims in published maps and institutional affiliations.

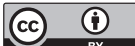

Open Access This article is licensed under a Creative Commons Attribution 4.0 International License, which permits use, sharing, adaptation, distribution and reproduction in any medium or format, as long as you give appropriate credit to the original author(s) and the source, provide a link to the Creative Commons license, and indicate if changes were made. The images or other third party material in this article are included in the article's Creative Commons license, unless indicated otherwise in a credit line to the material. If material is not included in the article's Creative Commons license and your intended use is not permitted by statutory regulation or exceeds the permitted use, you will need to obtain permission directly from the copyright holder. To view a copy of this license, visit http://creativecommons.org/ licenses/by/4.0/.

(C) The Author(s) 2018 